\title{
Erratum: High-harmonic generation from hydrogen atoms driven by two-color mutually orthogonal laser fields [Phys. Rev. A 88, 063419 (2013)]
}

\author{
Mitsuko Murakami, Oleg Korobkin, and Marko Horbatsch \\ (Received 5 May 2017; published 23 May 2017)
}

DOI: 10.1103/PhysRevA.95.059909

It was brought to our attention that formulas (15) and (18) in our paper contain a spurious centrifugal term. The correct formulas are as follows:

$$
\begin{gathered}
\left\langle a_{z}\right\rangle=-\sum_{\ell, m, j} \frac{c_{\ell}^{m}}{r^{2}\left(x_{j}\right)} \operatorname{Re}\left[\varphi_{\ell+1}^{m *}\left(x_{j}\right) \varphi_{\ell}^{m}\left(x_{j}\right)\right], \\
\left\langle a_{x}\right\rangle=-\frac{1}{2} \sum_{\ell, m, j} \frac{1}{r^{2}\left(x_{j}\right)} \operatorname{Re}\left[\kappa_{\ell}^{m} \varphi_{\ell}^{m *}\left(x_{j}\right) \varphi_{\ell+1}^{m+1}\left(x_{j}\right)+\kappa_{\ell}^{-m} \varphi_{\ell}^{m *}\left(x_{j}\right) \varphi_{\ell+1}^{m-1}\left(x_{j}\right)\right] .
\end{gathered}
$$

We acknowledge S. Brennecke for finding these errors. The relative difference in accelerations due to these errors is on the level of $10^{-8}$, which is negligible for any of the results reported in our paper. 